\title{
Lacosamide and Epilepsy
}

\author{
Jacklyn A. Harris \& Julie A. Murphy \\ Department of Pharmacy Practice, St. Louis College of Pharmacy, St. Louis, MO, USA
}

\author{
Keywords \\ Antiepileptic drug; Epilepsy; Lacosamide; \\ Partial-onset seizures. \\ Correspondence \\ Jacklyn A. Harris, PharmD, BCPS, Department \\ of Pharmacy Practice, St. Louis College of \\ Pharmacy, 4588 Parkview Place, St. Louis, \\ MO 63110-1088, USA. \\ Tel.: +314-446-8542; \\ Fax: +314-446-8500; \\ E-mail: jharris2@stlcop.edu
}

\begin{abstract}
SUMMARY
We will review all available studies on the use of lacosamide in the treatment of partial-onset seizures. The available evidence includes two open-label studies and three randomized controlled trials evaluating the safety and efficacy of oral lacosamide. One open-label study and one randomized controlled trial evaluating the safety and tolerability of intravenous lacosamide was also identified. Lacosamide was found to be efficacious with significant reduction in seizure frequency dosed 400-600 mg daily. Moreover, its adverse drug effects were mild and infrequently reported in the literature. Findings suggest that lacosamide is an effective agent for adjunctive treatment of refractory partial-onset seizures.
\end{abstract}

\section{Introduction}

There are three clinical types of partial seizures including simple partial, complex partial, and partial seizure evolving into secondarily generalized seizures. A task force of individuals from the American Academy of Neurology and the American Epilepsy Society provided evidence-based recommendations for the treatment of partial seizures $[1,2]$. In general, carbamazepine is considered firstline therapy for partial-onset seizures. However, less than $50 \%$ of patients will remain seizure-free after beginning initial antiepileptic drug (AED) therapy [3]. If carbamazepine fails to control the seizures, lamotrigine, topiramate, tiagabine, gabapentin, levetiracetam, oxcarbazepine, pregabalin, and zonisamide may be either added to carbamazepine or used as monotherapy. Although these agents have demonstrated similar efficacy for the treatment of partial-onset seizures, they are not always well tolerated by patients. In fact, $20-30 \%$ of patients will discontinue therapy because of intolerable adverse drug effects (ADEs) [4]. Based upon the percentage of patients with epilepsy who are refractory to or unable to tolerate AEDs due to ADEs, it is evident that effective AEDs with minimal adverse drug reactions are needed. The manufacturers of lacosamide (other names include harkoseride, SPM-927, and ADD-234037) are hoping to assist in meeting this need. Lacosamide, unlike other AEDs, enhances the slow inactivation of voltage gated sodium channels (VGSCs) and interacts with the collapsin-response mediator protein-2 (CRMP-2) [5-7]. With the addition of this novel mode of action, lacosamide may have a role as adjunctive treatment of partial-onset seizures with minimal ADEs. This review explores the role of lacosamide in the management of partial-onset seizures.

\section{Lacosamide}

\section{Pharmacology}

Lacosamide (R-2-acetamido- $N$-benzyl-3-methoxypropionamide) is a functionalized amino acid molecule believed to have a dual mode of action. It has been postulated that lacosamide selectively enhances the slow inactivation of VGSCs [5] and interacts with CRMP-2 [6]. Lacosamide shifts the slow inactivation curve to a more hyperpolarized potential, thereby selectively enhancing the slow inactivation of VGSCs. In return, this allows for more membrane potentials to enter the slow inactivation state prolonging depolarization. By prolonging depolarization, there are a limited number of neurons available for depolarization. This may ultimately lead to a decrease spread of a partial seizure. Unlike many other AEDs, lacosamide does not affect fast inactivation [5-7].

Lacosamide has been shown to interact with CRMP-2. It is not known what effect CRMP-2 has on seizure control. However, a study examining the hippocampus of patients with drug-resistant mesial temporal lobe epilepsy found reduced levels of CRMP-2 expressed compared to the control patients without epilepsy [8]. 
Lacosamide has been shown to interfere with CRMP-2 mediated neurotrophic effects, including axonal outgrowth [6]. Although the exact mechanism is not fully understood, this novel mechanism of interacting with CRMP-2 may be beneficial to patients with refractory seizures.

\section{Pharmacokinetics}

Lacosamide is rapidly and completely absorbed following oral administration displaying linear pharmacokinetics, with negligible first pass effect [9-11]. The bioavailability of lacosamide is approximately $100 \%$, without regard to food. Intravenous (IV) lacosamide demonstrates bioequivalence when infused over 30 or $60 \min [9,10]$.

Following oral administration, peak serum concentrations of lacosamide occur in approximately $1-4 \mathrm{~h}$, with a plasma half-life of about $13 \mathrm{~h}$ [9-11]. Lacosamide is metabolized to its inactive metabolite, O-desmethyl metabolite, which has not been found to have any pharmacological activity. About $30 \%$ of the O-desmethyl metabolite and $40 \%$ of the unchanged lacosamide dose are renally eliminated.

Because lacosamide has minimal protein binding $(<15 \%)$, the risk for drug-drug interactions is low. Although lacosamide was found to inhibit CYP-450-2C19 in in vitro studies at concentrations greater than 15 times higher than therapeutic plasma levels, no clinically relevant effects on plasma concentrations were found when lacosamide was given to poor or extensive metabolizers of CYP-2C19. Lacosamide has no effect on the serum levels of other AEDs, including carbamazepine, levetiracetam, lamotrigine, topiramate, valproate, zonisamide, gabapentin, and phenytoin. Other drug interaction studies have also found no effect on the pharmacokinetics of metformin, digoxin, oral contraceptives, and omeprazole [9-11].

\section{Role in Epilepsy \\ Oral Lacosamide}

Two open-label, multicenter studies were performed to investigate the safety and tolerability of lacosamide as adjunctive therapy in subjects with refractory partial seizures $[12,13]$. Eleven of the 13 subjects $(84 \%)$ in the study by Fountain et al. were titrated to the maximal daily dose of $600 \mathrm{mg}$ [12]. In the study by Sachdeo et al., 100 subjects were enrolled in the study, however data for analysis was only available for 90 subjects [13]. The median maximum tolerated dose was $300 \mathrm{mg} /$ day with $40 \%$ of subjects having a maximum tolerated dose of $500 \mathrm{mg}$ or $600 \mathrm{mg}$ daily. It is unknown what other AEDs were used by the subjects in these two studies.

In a multicenter, double-blind, placebo-controlled trial, the safety and efficacy of lacosamide $400 \mathrm{mg}$ daily, and $600 \mathrm{mg}$ daily when added to concomitant AED therapy in 405 subjects with uncontrolled partial-onset seizures was evaluated [14]. During the baseline phase of the study, if subjects experienced at least eight seizures with no more than a 21-day seizure-free period, they were randomized in a 1:2:1 ratio to placebo, lacosamide $200 \mathrm{mg}$ twice daily or lacosamide $300 \mathrm{mg}$ twice daily, respectively. Concomitant AEDs were held stable throughout the trial. The lacosamide dose was titrated by $100 \mathrm{mg}$ daily increments per week to the randomized dose. The median percent reduction in seizure frequency (maintenance vs. baseline) per 28 days, using intentto-treat analysis, was $20.8 \%, 37.3 \%(P=0.0078)$, and $37.8 \%$ $(P=0.0061)$ for the placebo, $400 \mathrm{mg} /$ day, and $600 \mathrm{mg} /$ day groups, respectively. The $50 \%$ responder rates were $18.3 \%, 38.3 \%$, and $41.2 \%$ for the placebo, $400 \mathrm{mg} /$ day, and $600 \mathrm{mg} /$ day groups, respectively. Only subjects completing the maintenance period with complete efficacy data were included in the seizure-free status analysis. The percentage of subjects that were seizure-free during the trial was $2.5 \%$ (400 mg daily) and $8.1 \%$ (600 mg daily). It is unknown what other AEDs were used by the subjects. The authors concluded that adjunctive lacosamide significantly reduced seizure frequency in patients with partial-onset seizures and was generally well tolerated.

In a multicenter, multinational, double-blind, placebocontrolled, randomized trial, the efficacy and safety of lacosamide 200, 400, and $600 \mathrm{mg}$ daily in two equally divided doses when added to concomitant AED therapy in subjects with uncontrolled partial-onset seizures was evaluated [15]. During the baseline period, subjects must have had at least four partial seizures per 28 days on average, with no seizure-free period longer than 28 days. For subjects randomized to $600 \mathrm{mg}$ daily, lacosamide was titrated in $100 \mathrm{mg}$ daily increments each week for 6 weeks. Subjects randomized to $400 \mathrm{mg}$ daily received placebo during the first 2 weeks of titration, then were started on lacosamide $100 \mathrm{mg}$ daily at week 3 and the dose was increased by $100 \mathrm{mg}$ daily increments each week until the randomized dose was reached. Subjects randomized to $200 \mathrm{mg}$ daily received placebo during the first 4 weeks of titration, then were started on lacosamide $100 \mathrm{mg}$ daily at week 5, with the dose being increased to $200 \mathrm{mg}$ daily during week 6 . Four hundred eighteen subjects were analyzed for safety and 415 subjects were included in the efficacy analysis. The median percent reduction in seizure frequency (maintenance vs. baseline) per 28 days, using intent-to-treat analysis, was $10 \%$, $26 \%, 39 \%(P=0.023)$, and $40 \%(P=0.0084)$ for the placebo, $200 \mathrm{mg} /$ day, $400 \mathrm{mg} /$ day, and $600 \mathrm{mg} /$ day groups, respectively. The $50 \%$ responder rates were $21.9 \%, 32.7 \%(P=0.899)$, $41.1 \%(P=0.0038)$, and $38.1 \%(P=0.0141)$ for the placebo, $200 \mathrm{mg} /$ day, $400 \mathrm{mg} /$ day, and $600 \mathrm{mg} /$ day groups, respectively. Seven subjects $(1.7 \%)$ remained seizure-free during the trial. The dose that these subjects were receiving is unknown. It is also unknown what other AEDs were used by the subjects. The authors concluded that adjunctive lacosamide significantly reduced seizure frequency in patients with partial-onset seizures.

A multicenter, double-blind, placebo-controlled, randomized trial was performed to evaluate the efficacy and safety of lacosamide (200 and $400 \mathrm{mg}$ daily) when added to one to three concomitant AEDs in 477 subjects with uncontrolled partial-onset seizures [16]. Subjects were to have had at least four partial seizures per 28 days on average, with no seizure-free period longer than 28 days during the period before enrollment as well as during the baseline period. For subjects randomized to $400 \mathrm{mg}$ daily, lacosamide was titrated in $100 \mathrm{mg}$ daily increments weekly. Subjects randomized to $200 \mathrm{mg}$ daily received placebo during the first 
2 weeks of titration, then were started on lacosamide $100 \mathrm{mg}$ daily at week 3 and the dose was increased to $200 \mathrm{mg}$ daily at week 4 of the titration period. The median percent reduction in seizure frequency (maintenance vs. baseline) per 28 days was $20.5 \%, 35.3 \%$ $(P=0.02)$, and $36.4 \%(P=0.03)$ for the placebo, $200 \mathrm{mg} /$ day, and $400 \mathrm{mg} /$ day groups, respectively. The $50 \%$ responder rates were $25.8 \%, 35 \%(P=0.07)$, and $40.5 \%(P=0.01)$ for the placebo, $200 \mathrm{mg} /$ day, and $400 \mathrm{mg} /$ day groups, respectively. The percentage of subjects that were seizure-free during the trial was $2.1 \%$ (placebo), 3.6\% (200 mg daily), and $2.4 \%$ (400 mg daily). It is unknown what other AEDs were used by the subjects. The authors concluded that adjunctive lacosamide significantly reduced seizure frequency in patients with partial-onset seizures and was generally well tolerated.

\section{Lacosamide}

A multicenter, double-blind, double-dummy, randomized trial was performed to evaluate the safety and tolerability of IV lacosamide as replacement therapy for oral lacosamide [17]. Subjects who were stable on twice daily dosing of oral lacosamide (200-600 mg daily) were randomized in a 2:1 ratio to one of two treatment groups: (1) IV lacosamide and placebo tablets twice daily or (2) IV placebo and oral lacosamide tablets twice daily. The first 30 subjects enrolled received the infusion over $60 \mathrm{~min}$, to allow for safety data evaluation, before the next 30 subjects received a 30-min infusion. Subjects who received at least one dose of trial medication were included in the primary safety analysis.

Fifty-nine of the 60 subjects enrolled completed the trial. Eighty percent of subjects were seizure-free during the trial. The daily number of seizures experienced by subjects in this trial remained stable compared to baseline. The authors concluded that IV lacosamide, administered as $60-$ or 30 -min twice daily infusions, showed a similar safety and tolerability profile to oral lacosamide for short-term replacement therapy. The percentage of subjects that had a $50 \%$ response rate was not reported.

A multicenter, open-label, inpatient trial was conducted to investigate whether IV lacosamide was safe and well tolerated when given twice daily as short-term adjunctive therapy in 160 subjects with partial seizures [18]. The investigators also attempted to identify appropriate infusion rate(s) for IV lacosamide. Subjects who were stable on twice daily dosing of oral lacosamide (200-800 mg daily) were placed into one of four cohorts: 30min infusion $(n=40), 15$-min infusion $(n=41)$, 10-min infusion $(\mathrm{n}=20)$, and final 15-min infusion $(\mathrm{n}=59)$. Subjects were given IV lacosamide twice daily for 2-5 days, per the discretion of the investigator and subject. Ninety-nine percent of subjects received at least 2 days of IV lacosamide twice daily and $53 \%$ of subjects in the 15-min and 10-min infusion groups received 3-5 days of therapy. The total daily dose received by subjects was 200-400 mg (61\%), 500-600 mg (35\%), or 700-800 mg (4\%). Infusion site related adverse events were infrequently reported, vital signs remained stable, and seizure patterns for individual subjects did not change. The percentage of subjects that were seizure-free or had a $50 \%$ response rate were not reported. Seventy-two percent of subjects were treated with one or two concomitant AEDs, most commonly levetiracetam, carbamazepine, lamotrigine, or valproate.

\section{Role in Status Epilepticus}

One case report describing the successful use of lacosamide administered via percutaneous gastrostomy tube for the treatment of convulsive status epilepticus was published [19]. Temporary interruption of the seizures was achieved after administration of $150 \mathrm{mg}$ (minced tablet powder) of lacosamide via gastostomy tube. Complete clinical remission of the epileptic activity was achieved within $30 \mathrm{~min}$ of administration of a second $150 \mathrm{mg}$ lacosamide dose (syrup) via gastrostomy tube. A second case report, describing the successful use of IV lacosamide for the treatment of nonconvulsive status epilepticus, was published [20]. A 200-mg bolus was administered without dilution, and within 3-5 min, rhythmic epileptiform activity ceased. The patient recovered from her symptoms completely.

Lacosamide could be a treatment alternative if other, more established medications fail or are considered inappropriate. The authors of both case reports conclude that large, prospective studies are needed to assess the safety and efficacy of lacosamide in status epilepticus.

\section{Adverse Effects}

The adverse effects that occurred in at least $5 \%$ of subjects in at least one of the clinical trials discussed are summarized in Table 1 [14-18].

Data from the IV lacosamide open-label trials, suggest that lacosamide IV is overall well tolerated. Dizziness occurred in 5\% and $6 \%$ of subjects receiving the IV lacosamide as 10- and 15-min infusions, respectively [18]. Dizziness, injection site pain, and somnolence was associated with the 30-min infusion, whereas headache, back pain, and dizziness was associated with the 60-min infusion $[17,18]$. Only minimal changes in the mean QTc interval and small increases in mean PR intervals were seen [17].

In a 6 month postmarketing experience, $52 \%$ (13/25) of patients reported side effects to lacosamide during the titration phase [21]. A sodium channel blocking anticonvulsant was used in addition to lacosamide in $70 \%$ of those patients reporting side effects. Most of the initial side effects did disappear during the

Table 1 Adverse effects associated with lacosamide [14-18]

\begin{tabular}{lc}
\hline Adverse effects & Subjects (\%) \\
\hline Abnormal coordination & 5.3 to $>10$ \\
Ataxia & 13 \\
Blurred vision & $>10$ \\
Diplopia & $9-12$ \\
Dizziness & $6-35$ \\
Fatigue & $5.6-14$ \\
Headache & $7-16$ \\
Nausea & $6.8-14$ \\
Nystagmus & 6 to $>10$ \\
Somnolence & $>10$ \\
Tremor & $>10$ \\
Upper respiratory tract infection & 10 \\
Vertigo & 6.5 \\
Vomiting & $4.3-12$ \\
\hline
\end{tabular}


maintenance phase or with dose reduction. Two patients lost more than $10 \%$ of their body weight while receiving lacosamide, but they were also receiving other AEDs (zonisamide, lamotrigine, and levetiracetam). Three patients discontinued therapy as a result of intolerable side effects. The most common reported ADEs were fatigue, double vision, depression, dizziness, and nausea.

\section{Contraindications/Precautions}

There are no documented contraindications to lacosamide. Like other AEDs, lacosamide could potentially increase the risk of suicidal thoughts or behaviors [22]. In addition, lacosamide should be withdrawn over at least 1 week to decrease the potential of increase seizure frequency. Patients receiving lacosamide should be advised that it may cause dizziness and ataxia. Dizziness was one of the most commonly reported adverse effects and reason for discontinuation in clinical trials. Lacosamide should also be used in caution in patients with severe cardiac disease and patients who are receiving medications known to induce PR interval prolongation. It is recommended that patients with severe cardiac diseases obtain an ECG before beginning lacosamide and after titrated doses to steady state. Although no cases of atrial fibrillation or flutter were reported in epilepsy trials, studies in diabetic neuropathy revealed $0.5 \%$ of patients developed atrial fibrillation or flutter. Syncope was only reported in patients with diabetic neuropathy as well. Patients developing syncope were receiving doses greater than $400 \mathrm{mg} /$ day of lacosamide. There is a risk for multiorgan hypersensitivity reactions when administering lacosamide. There has been one case of a patient developing symptomatic hepatitis and nephritis during initial clinical studies consistent with multiorgan hypersensitivity. Three other potential cases include two patients who developed a rash and elevated liver enzymes, and one patient who developed myocarditis and hepatitis.

\section{Dosage and Administration}

Lacosamide should be initiated at $50 \mathrm{mg}$ twice daily for the adjunctive treatment of partial-onset seizures [22]. The dose can be increased weekly by $100 \mathrm{mg}$ daily given as two divided doses, up to the recommended total daily dose of 200 or $400 \mathrm{mg}$. The Food and Drug Administration did not approve the $600 \mathrm{mg}$ total daily dose, due to no additional benefit and the increased risk of additional side effects over a $400 \mathrm{mg}$ total daily dose [22]. Patients receiving IV lacosamide can receive a dosage equivalent to their oral lacosamide dose. The IV dose should be infused over 30-60 min.

For patients with severe renal impairment, defined as a creatinine clearance of less than $30 \mathrm{~mL} / \mathrm{min}$, the maximum daily recommended dose is $300 \mathrm{mg}$ [22]. A supplement dosage of up to $50 \%$ should be considered following a 4-h hemodialysis treatment. The maximum daily recommended dose for patients with mild or moderate hepatic impairment is $300 \mathrm{mg}$. It is recommended to exercise caution when increasing the dosage of lacosamide in patients with renal or hepatic impairment. Efficacy and safety of lacosamide in patients under 17 years old has not been established.

Lacosamide is available as $50 \mathrm{mg}, 100 \mathrm{mg}, 150 \mathrm{mg}$, and $200 \mathrm{mg}$ tablets [22]. It is also available in a $200 \mathrm{mg} / 20 \mathrm{~mL}$ single dose vial, which is stable at room temperature. The injection may be injected without further dilution and is compatible with sodium chloride injection $0.9 \%$, lactated ringers, and dextrose.

\section{Conclusions}

Despite the limited number of placebo-controlled trials evaluating the safety and efficacy of lacosamide as adjunctive treatment for partial-onset seizures, the data suggests that lacosamide not only consistently reduces seizure frequency at doses ranging from 400 to $600 \mathrm{mg}$ daily, but is also well tolerated. Lacosamide's pharmacokinetic profile minimizes the risk of drug interactions. These factors, in addition to its novel mechanism of action, make lacosamide an attractive alternative to other approved AEDs for the adjunctive treatment of partial-onset seizures.

Further research needs to be completed to define its role in status epilepticus refractory to other agents. Current literature supports the role of lacosamide adjunctive therapy for partial-onset seizures, however, the role of lacosamide as monotherapy needs to be established.

Lacosamide has also been studied for the treatment of diabetic neuropathic pain [23-31]. Although many studies in this area are promising, showing minimal improvement in clinical pain scores, additional randomized-controlled trials are necessary to determine its exact place in this condition.

\section{Author Contributions}

Both authors contributed equally to writing this review.

\section{Conflict of Interest}

The authors have no conflict of interest with any commercial or other associations in connection with the submitted article.

\section{References}

1. French JA, Kanner AM, Bautista J, et al. Efficacy and tolerability of the new antiepileptic drugs I: Treatment of new onset epilepsy: Report of the Therapeutics and Technology Assessment Subcommittee and Quality Standards Subcommittee of the AAN and the AES. Neurology 2004; 62:1252-1260.

2. French JA, Kanner AM, Bautista J, et al. Efficacy and tolerability of the new antiepileptic drugs II: Treatment of refractory epilepsy. Report of the Therapeutics and Technology Assessment Subcommittee and Quality Standards Subcommittee of the AAN and the AES. Neurology 2004;62:1261-1273.

3. Kwan P, Brodie MJ. Epilepsy after the first drug fails: Substitution or add-on? Seizure 2000;9:464-468,

4. Hovinga CA. SPM-927. IDrugs 2003;6:479-485.

5. Errington AC, Stohr T, Heers C, Lees G. The investigational anticonvulsant lacosamide selectively enhances slow inactivation of voltage-gated sodium channels. Mol Pharmacol 2008;73:157-169.

6. Beyreuther BK, Freitag J, Heers C, Krebsfänger N, Scharfenecker U, Stöhr T. Lacosamide: A review of preclinical properties. CNS Drug Rev 2007;13:21-42.

7. Beydoun A, D'Souza J, Hebert D, Doty P. Lacosamide: Pharmacology, mechanisms of action and pooled efficacy and safety data in partial-onset seizures. Expert Rev Neurother 2009;9:33-42.

8. Czech T, Yang JW, Csaszar E, Kappler J, Baumgartner C, Lubec G. Reduction of hippocampal collapsing response mediated protein-2 in patients with mesial temporal lobe epilepsy. Neurochem Res 2004;29:2189-2196.

9. Bialer M, Johannessen S, Levy R, Perucca E, Tomson T, White HS. Progress report on new antiepileptic drugs: A summary of the Ninth Eilat Conference (EILAT IX). Epilepsy Res 2009;83:1-42.

10. Doty P, Rudd GD, Stoehr T, Thomas D. Lacosamide. Neurotherapeutics 2007;4:145-148. 
11. Kellinghaus C. Lacosamide as treatment for partial epilepsy: Mechanisms of action, pharmacology, effects, and safety. Ther Clin Risk Manag 2009;5:757-766.

12. Fountain NB, French JA, Privitera MD. Harkoseride: Safety and tolerability of a new antiepileptic drug (AED) in patients with refractory partial seizures [abstract]. Epilepsic 2000;41(Suppl 7):169-170.

13. Sachdeo R, Montouris G, Beydoun A, et al. An open-label, maximum tolerated dose trial to evaluate oral SPM 927 as adjunctive therapy in patients with partial seizures [abstract]. Neurology 2003;60(Suppl 1):A433.

14. Chung SS, Sperling MR, Biton V, et al.; SP754 Study Group. Lacosamide as adjunctive therapy for partial-onset seizures: A randomized controlled tiral. Epilepsia 2010;51:958-967.

15. Ben-Menachem E, Biton V, Jatuzis D, Abou-Khalil B, Doty P, Rudd GD. Efficacy and safety of oral lacosamide as adjunctive therapy in adults with partial-onset seizures. Epilepsia 2007;48:1308-1317.

16. Halasz P, Kalviainen R, Mazurkiewicz-Beldzinska M, et al.; SP755 Study Group. Adjunctive lacosamide for partial-onset seizures: Efficacy and safety results from a randomized controlled trial. Epilepsia 2009;50:443-453.

17. Biton V, Rosenfeld WE, Whitesides J, Fountain NB, Vaiciene N, Rudd GD. Intravenous lacosamide as replacement for oral lacosamide in patients with partial-onset seizures. Epilepsia 2008;49:418-424.

18. Krauss G, Ben-Menachem E, Mameniskiene R, Vaiciene-Magistris N, Brock M, Whitesides JG, Johnson ME. Intravenous lacosamide as short-term replacement for oral lacosamide in partial-onset seizures. Epilepsia 2010;51:951-957.

19. Tilz C, Resch R, Hofer T, Eggers C. Successful treatment of refractory convulsive status epilepticus by non-parenteral lacosamide. Epilepsia 2010;51:316-317.

20. Kellinghaus C, Berning S, Besselmann M. Intravenous lacosamide as successful treatmen for nonconvulsive status epilepticus after failure of first-line therapy. Epilepsy Behav 2009; 14;429-431.
21. Wehner T, Bauer S, Hamer H, et al. Six months of postmarketing experience with adjunctive lacosamide in patients with pharmacoresistant focal epilepsy at a tertiary epilepsy center in Germany. Epilepsy Behav 2009;16:423-425.

22. Package insert. Vimpat (lacosamide tablets and injection). Smyrna, GA: UCB, Inc, January 2009

23. Shaibani A, Biton V, Rauck R, Koch B, Simpson J. Long-term oral lacosamide in painful diabetic neuropathy: A two-year open-label extension trial. Eur J Pain 2009;13:458-463.

24. Rauck RL, Shaibani A, Biton V, Simpson J, Koch B. Lacosamide in painful diabetic peripheral neuropathy: A phase 2 double-blind placebo-controlled study. Clin J Pain 2007:23:150-158.

25. McCleane G, Koch B, Rauschkolb C. A report of continuing analgesia after 12 months treatment with SPM 927 in six subjects with neuropathic pain. J Neuropathic Pain Symptom Palliation 2005; 1:25-28.

26. Bretschneider $M$, Bongardt S, Koch B. A multi-center, open-label, follow-on trial to assess the long-term safety and efficacy of lacosamide in subjects with painful diabetic neuropathy [abstract]. J Pain 2006;7:S43.

27. Hidvegi $T$, Bretschneider $M$, Thierfelder S, Sommerville $K$, Bongardt S. Long-term efficacy of lacosamide in subjects with painful distal diabetic neuropathy [abstract]. J Pain 2008;9:P27.

28. McCleane G, Koch B, Rauschkolb C. Does SPM 927 have an analgesic effect in human neuropathic pain? An open label study. Neurosci Lett 2003;352:117-120.

29. Graf R, Frye W, Simpson J. Lacosamide in long-term treatment of painful diabetic neuropathy (DNP) [abstract]. Eur J Neurol 2007; 14(Suppl 1):260.

30. Bongardt S, Shaibani A, Kenney P, Simpson J. Lacosamide in painful distal diabetic neuropathy: Results of a multi-center, placebo-controlled US trial [abstract]. Eur J Neurol 2007; 14(Suppl 1):250

31. Wymer JM, Garrison C, Simpson J, Koch B. A multicenter, randomized, double-blind, placebo-controlled trial assessing the efficacy and safety of lacosamide in painful distal diabetic neuropathy [abstract]. Ann Neurol 2006;60(Suppl 3):S68. 